\title{
Pectoralis Major Myocutaneous Flap; Indications and Limitations
}

The pectoralis major myocutaneous flap described by Ariyan has revolutionized reconstruction in head and neck surgery. The physiology and surgical anatomy of this dependable fiap are illustrated. The technique commonly used permits a simultaneous or subsequent ipsilateral deItopectoral fiap. Indications for the pectoralis major myocutaneous fiap are skin, mucosal and bone defects; necrosis of neck skin after radiation therapy and subsequent surgery; tongue defects ; hypopharyngeal and cervical esophageal reconstruction; augmentation after mandibular resection, particularly in anterior arch defects. Advantages are the one-stage reconstruction, the relative technical ease, the good cosmetic acceptance, the fiap viability, the opportunity to
Volker Jahnke, M. D., F. A. C. S.

Professor and Chairman, Department of Otolaryngology, Rudolf-Virchow-Krankenhaus Augustenburger Platz 11000 Berlin 65 West Germany

incorporate bone for mandibular reconstruction, the effective carotid artery protection, the avoidance of long hospitalization. Limitations are the thickness of the tissue transferred, the bulkiness of the muscle pedicle and -less subtantialthe transfer of hair bearing skin into the oral cavity in man and the breast distortion in women or the occasional problem with primary closure of the donor site. Though some of these disadvantages may be reduced or avoided, this method does not completely replace regional skin fiaps or free fiaps. Cases presentations demonstrate the successful use of the pectoralis major myocutaneous fiap for a variety of head and neck reconstructive procedures. 